\title{
A COMPOSIČÃO SOCIAL IMPORTA PARA OS EFEITOS DAS ESCOLAS NO ENSINO FUNDAMENTAL?
}

\author{
FLAVIA PEREIRA XAVIER \\ MARIA TERESA GONZAGA ALVES
}

* Agradecemos ao Conselho Nacional de Desenvolvimento Científico e Tecnológico (CNPq), à Fundação de Amparo à Pesquisa do Estado de Minas Gerais (FAPEMIG) e à Pró-Reitoria de Pesquisa da Universidade Federal de Minas Gerais (PRPq/ UFMG) pelo apoio a esta

\section{RESUMO}

O artigo analisa o efeito das escolas públicas brasileiras de ensino fundamental para o aprendizado de seus alunos, considerando $o$ contexto socioeconômico e a composição do alunado por gênero e raça. Os alunos foram distribuídos em três níveis de proficiência: insuficiente, básico e adequado. Os que estão no nível insuficiente são considerados excluídos, uma vez que o seu direito à educação não é atendido. Utilizaram-se os dados de 2007, 2009 e 2011 da Prova Brasil. Os principais resultados indicam que os efeitos das escolas para a retirada dos alunos da exclusão e para sua promoção ao nível adequado de aprendizagem estão associados às características de composição social dessas escolas em termos de raça e gênero, independentemente do nível socioeconômico médio da escola. Tal composição também afeta as chances individuais de os alunos serem retirados da exclusão e promovidos ao nível de adequação.

PALAVRAS-CHAVE EFEITOS DAS ESCOLAS • DESIGUALDADES ESCOLARES • NÍVEIS DE PROFICIÊNCIA • ENSINO FUNDAMENTAL. 


\section{RESUMEN}

El artículo analiza el efecto de las escuelas públicas brasileñas de educación básica para el aprendizaje de sus alumnos, considerando el contexto socioeconómico y la composición del alumnado por género y raza. Los alumnos se distribuyeron en tres niveles de competencia: insuficiente, básico y adecuado. Se consideran excluidos los que están en el nivel insuficiente, una vez que su derecho a la educación no es atendido. Se utilizaron datos de 2007, 2009 y 2011 de la Prova Brasil. Los principales resultados indican que los efectos de las escuelas para retirar los alumnos de la exclusión y promoverlos al nivel adecuado de aprendizaje están asociados a las características de composición social de dichas escuelas en lo que concierne a raza y género, independientemente del nivel socioeconómico promedio de la escuela. Tal composición también afecta las oportunidades individuales de que los alumnos sean retirados de la exclusión y promovidos al nivel de adecuación.

PALABRAS CLAVE EFECTO DE LAS ESCUELAS •DESIGUALDADES ESCOLARES • NIVELES DE COMPETENCIA • EDUCACIÓN BÁSICA.

\section{ABSTRACT}

This article analyzes the effect of Brazilian public elementary schools on students' learning considering the socio-economic context and students' gender and racial composition. Students were allocated at three levels of proficiency: insufficient, basic and adequate. Those at the insufficient level are considered excluded since their right to education is not met. The data used was from the 2007, 2009 and 2011 Prova Brasil. The main results indicate that the effects of the schools to remove students from exclusion and promote them to adequate levels of learning are associated with the social composition of these schools in terms of race and gender, regardless of the average socioeconomic status of the school. Such composition, also affects the chances of individual students being removed from exclusion and promoted to the adequate level.

KEYWORDS SCHOOL EFFECTS - SCHOOL INEQUALITY • PROFICIENCY LEVELS • ELEMENTARY EDUCATION. 
1 Status pode ser entendido como um conjunto de direitos e obrigações que especificam as atividades esperadas dos indivíduos incumbidos de uma posição social (HALLER; SARAIVA, 1991).

2 A visão otimista atribuía à educação um papel central no duplo processo de superação do atraso econômico e dos privilégios adscritos

- sociedades tradicionais - e da formação de uma nova sociedade moderna, meritocrática e democrática. É a visão dominante até meados do século $X X$
Em oposição à corrente predominante dos estudos até meados do século XX, que atribuía à escola um papel essencial à alocação de status ${ }^{1}$ como promotora da meritocracia (PARSONS, 1970, 1974; DAVIS; MOORE, 1981), a pesquisa sociológica a partir de 1960 evidencia a estreita relação entre desigualdades sociais e o sucesso escolar (BOURDIEU; PASSERON, 1975; COLEMAN et al., 1966). Em outras palavras, esses estudos contribuíram para a visão pessimista da educação de que a "escola não faz a diferença" em oposição à visão otimista até então dominante, ${ }^{2}$ revelando a forte influência da origem familiar dos alunos para o seu destino escolar e ocupacional.

A qualidade da instrução, da infraestrutura e da gestão é condição que diferencia as escolas e poderia compensar, ou pelo menos minimizar, o determinismo social, sobretudo para os alunos de origem social desfavorecida (RUTTER et al., 1979; MORTIMORE et al., 2008; WILLMS, 1992). O estudo dessas diferenças levou ao desenvolvimento de um campo de pesquisas denominado, na literatura, como efeito-escola (LEE, 2001). 
No Brasil, as pesquisas sobre o efeito das escolas se desenvolveram, a partir dos anos 1990, com a disponibilidade de dados produzidos pelas avaliações educacionais em larga escala, que, pela primeira vez, reuniram informações sobre aprendizado dos alunos, condições de escolarização e características dos alunos (ALBERNAZ; FERREIRA; FRANCO, 2005; ANDRADE; LAROS, 2007; BARBOSA; FERNANDES, 2001; FLETCHER, 1997; SOARES; CÉSAR; MAMBRINI, 2001; entre outros). As avaliações nacionais revelaram que a universalização do acesso à educação não estava garantindo o aprendizado dos estudantes. Esses resultados, congruentes com os estudos internacionais, eram explicados, sobretudo, pelas características socioeconômicas do alunado, portanto, fora do controle da escola (ALVES; FRANCO, 2008).

Apesar da crescente influência das informações das avaliações em larga escala para formulação e implantação das políticas públicas voltadas à melhoria do desempenho dos alunos (BROOKE; CUNHA, 2011), a educação brasileira parece viver um paradoxo. Por um lado, o monitoramento propiciou a implantação de políticas focalizadas, ${ }^{3}$ que contribuíram para melhoria no desempenho médio dos alunos brasileiros, conforme divulgado amplamente pela imprensa nacional.

Por outro lado, a associação entre as características socioeconômicas e demográficas dos alunos e o desempenho médio tem revelado um padrão persistente ao longo dos anos. Os estudantes das escolas privadas, que são brancos, possuem um nível socioeconômico mais alto e têm uma trajetória escolar regular, apresentam resultados médios sistematicamente melhores. Além disso, pouco se alterou o percentual de escolas que possuem práticas internas que levam seus alunos a resultados melhores do que era esperado (elevado efeito-escola), comparando-se com escolas com características semelhantes (SOARES; ALVES, 2013; ANDRADE; SOARES, 2008). Ou seja, a educação brasileira não consegue atingir objetivos equitativos ao mesmo tempo em que o desempenho médio geral avança.

Esse fenômeno pode ser descrito como uma translação da estrutura de desempenho escolar para o alto, em analogia
3 Entre essas políticas, destacam-se: criação da Prova Brasil, em 2005, que faz uma avaliação censitária das escolas públicas; introdução, pelo governo federal, do Índice de Desenvolvimento da Educação (Ideb), em 2007; e políticas de responsabilização que se disseminaram em vários estados brasileiros (BROOKE; CUNHA, 2011) 
à análise feita por Bourdieu (1998) sobre a expansão da escolarização em seu país, na segunda metade do século XX, quando a melhoria dos níveis educacionais da população em geral não alterou a ordem, as distâncias e diferenças entre as classes sociais. No caso do Brasil, como o desempenho dos alunos é, em geral, bastante baixo, há muito espaço para crescimento das médias, o que acaba ofuscando a diferença entre os grupos sociais (SOARES; MAROTTA, 2009).

Uma possível explicação para esse paradoxo reside na adoção da média do desempenho escolar como a principal medida de resultados. A média é uma síntese estatística muito sensível a valores extremos. Em decorrência, em países com elevadas desigualdades, é muito provável que o desempenho médio dos alunos nas avaliações esteja refletindo os melhores resultados de grupos de alunos em algumas escolas. Assim, o crescimento nas médias das proficiências pode mascarar o fato de que grande parte dos estudantes não tem o desempenho em nível adequado para o ano escolar em que se encontram. Isso é mais grave quando os resultados ruins associam-se a alguns grupos sociais. Por essa razão, é necessário descrever as desigualdades entre esses grupos e escolas com mais reflexão e transparência.

Tendo em vista a compreensão das desigualdades escolares no Brasil, evidenciando o seu padrão de distribuição e os mecanismos que as reforçam, pretende-se responder às seguintes questões: qual o efeito das escolas em tirar os alunos dos níveis mais baixos da escala de proficiência para os níveis mais elevados? Esses efeitos são diferentes segundo o contexto socioeconômico e a composição dessas escolas? Qual o impacto do contexto e da composição das escolas nas chances de os seus alunos permanecerem numa situação de exclusão ou no nível de aprendizagem adequado?

\section{ABORDAGEM ANALÍTICA}

Para responder às questões deste artigo, utilizam-se como referência os estudos sobre efeito-escola e os fatores associados ao desempenho escolar. Esse campo de pesquisa constituiu-se em torno dos estudos que punham em dúvida a ideia de que 
as escolas não fazem diferença, sustentada pelas conclusões do Relatório Coleman, da década de 1960, e por outros trabalhos semelhantes (BROOKE; SOARES, 2008).

Sem negar o impacto dos fatores extraescolares, principalmente a origem social dos alunos, Rutter e colaboradores (1979) argumentavam que, se as escolas explicarem de $20 \%$ a $25 \%$ da variação no desempenho dos alunos, isso não deve ser negligenciado tendo em vista o tempo que o aluno fica na escola. Algumas escolas têm mais impacto do que outras, e isso pode fazer uma grande diferença para os alunos.

Willms (1992) demonstrou que o efeito das características dos alunos no desempenho escolar é potencializado pelo contexto escolar - definido pela composição social dos alunos -, que resulta na distribuição desigual de oportunidades educacionais. Assim, somente com o controle das características dos alunos e do contexto escolar é possível mensurar o efeito das escolas (RAUDENBUSH; WILLMS, 1995).

A literatura com base em dados brasileiros indica que o efeito do contexto escolar é particularmente forte no país. Nos estudos nacionais, esse efeito é mensurado pelo nível socioeconômico médio das escolas. Assim, a evidência é de que as escolas reproduzem no seu contexto a desigualdade social do país, tornando-as fortemente segregadas (ANDRADE; LAROS, 2007; ANDRADE; SOARES, 2008).

Os efeitos de composição social das escolas estão diretamente relacionados à influência dos pares. Isto é, estudantes com altos resultados e motivação podem ajudar a criar uma cultura de sucesso na escola, enquanto aqueles com baixo desempenho e motivação podem criar um ambiente de privação e desespero (RUMBERGER; PALARDY, 2005).

Entre os fatores que caracterizam o contexto escolar, um tema ainda pouco estudado refere-se às proporções de meninas e de alunos pretos por escola. A literatura mostra que as meninas possuem resultados menos favoráveis em Matemática, mas a presença maior destas na escola afeta positivamente a média de desempenho de todos os alunos, fato que pode estar ligado à criação de um ambiente mais disciplinado e favorável ao aprendizado (SOARES; ALVES, 2013). Carvalho (2003) faz uma reflexão das principais explicações 
4 Os trabalhos citados neste parágrafo utilizaram como referência empírica os dados do Sistema de Avaliação da Educação Básica - Saeb, que até 2003 adotava a seguinte nomenclatura para autoclassificação de cor ou raça: branco, pardo negro, amarelo e indígena. A partir de 2005, o Saeb passou a adotar a nomenclatura do Instituto Brasileiro de Geografia e Estatística (IBGE) substituindo negro por preto. sobre as diferenças dos indicadores educacionais entre gêneros no Brasil.

Os alunos negros também têm desempenho pior do que os brancos e pardos. Porém, em sentido oposto ao observado em relação à presença de meninas na escola, a maior proporção de alunos negros afeta negativamente a média de desempenho da escola (ANDRADE; LAROS, 2007). Em estudo realizado com dados nacionais, Soares e Alves (2003) demonstraram que as diferenças entre alunos negros e brancos acentuam-se à medida que as escolas passam a dispor de melhores condições de funcionamento. A equidade racial é um cenário mais provável nas piores escolas, o que não é um resultado pretendido. Ou seja, a qualidade da educação - medida pela média de desempenho do conjunto das escolas - e a equidade - a ausência de viés racial, de gênero, social em educação - são objetivos distintos. ${ }^{4}$

Mais recentemente, esse fenômeno passou a ser investigado pela análise da exclusão intraescolar. Essa noção caracteriza a situação do aluno matriculado na escola, mas cujo desempenho escolar é insuficiente para a série que ele cursa. A exclusão intraescolar não ocorre simplesmente pela dificuldade de acesso ao sistema escolar ou pela evasão deste. Ela informa sobre a ausência de aprendizado dentro da escola (SOARES et al., 2012).

O ponto central reside no fato de que os níveis insuficientes de aprendizagem não estão aleatoriamente distribuídos. Nesse sentido, espera-se que os grupos com maiores desvantagens socioeconômicas, culturais e de trajetória escolar irregular estejam sobrerrepresentados nos níveis mais baixos e sub-representados nos grupos de desempenho mais elevado. Do mesmo modo, espera-se que, nas escolas com sobrerrepresentação de grupos em desvantagem social e econômica, a composição dessas escolas afete negativamente o desempenho do conjunto dos seus alunos.

Analisar os resultados segundo níveis de desempenho é uma forma alternativa à abordagem convencional que estuda o efeito de fatores individuais e escolares sobre a proficiência média dos alunos. A grande vantagem dessa abordagem é que ela permite analisar a distribuição dos resultados em 
toda a escala de proficiência, atribuir sentido normativo a intervalos da escala de acordo com uma interpretação pedagógica apropriada para cada nível e evidenciar situações de exclusão (SOARES, 2009). A média da proficiência pode esconder situações de exclusão, pois é possível que a média de desempenho de uma escola ou de um sistema melhore pelo aumento da proficiência dos alunos mais capazes e não necessariamente pela diminuição das desigualdades entre os alunos. A exclusão educacional relativa ao desempenho tem consequências perversas para a aquisição educacional do aluno, principalmente o alcance aos níveis mais altos de ensino e ao acesso aos melhores centros, e para a entrada qualificada deste no mercado de trabalho.

Portanto, este trabalho inova ao tratar tanto os efeitos das escolas quanto a estimação dos impactos da composição escolar por níveis de desempenho.

\section{METODOLOGIA}

\section{DADOS}

Foram utilizados os dados de 2007, 2009 e 2011 da Prova Brasil, ${ }^{5}$ que é uma avaliação censitária com o objetivo de avaliar a qualidade do ensino ministrado nas escolas públicas. Nesses três ciclos, os alunos do $5^{\circ}$ e $9^{\circ}$ anos do ensino fundamental das escolas públicas urbanas e rurais com no mínimo 20 alunos fizeram testes de Leitura e Matemática e responderam a um questionário contextual, com informações demográficas, socioeconômicas e culturais, trajetória escolar e hábitos de estudo. Foram aplicados também questionários contextuais para professores, diretores e escolas, este último preenchido pelo aplicador do teste. ${ }^{6}$

O desempenho dos alunos na Prova Brasil é expresso em uma escala de proficiência, construída em 1997, que expressa os escores dos alunos com valores de 0 a 500 pontos. Como a escala é a mesma para os diferentes anos, pode-se assumir que as variações na proficiência dos alunos de determinado ano escolar, ao longo dos diferentes ciclos de aplicação, refletem a melhoria ou a piora no aprendizado das coortes avaliadas. Além disso, como a mesma escala é usada para

5 Os microdados da Prova Brasil estão disponíveis para download em $<$ http://portal.inep.gov.br/basicalevantamentos-acessar>. 
expressar os escores dos alunos dos diferentes anos escolares, os alunos do $5^{\circ}$ ano têm proficiências com valores mais baixos do que os do $9^{\circ}$ ano.

As análises apresentadas neste trabalho foram feitas tomando-se como resultado dos alunos apenas a respectiva proficiência em Matemática. A opção por essa disciplina justifica-se pelo fato de estudos brasileiros indicarem que o aprendizado dessa área é mais relacionado ao ambiente escolar do que a Leitura, que se beneficia mais do ambiente familiar (BARBOSA; FERNANDES, 2001).

Além disso, este trabalho inclui apenas os alunos das escolas estaduais e municipais. Essas redes respondem, juntas, por $86 \%$ das matrículas no ensino fundamental da educação básica, segundo dados do Censo Escolar de 2011. Finalmente, foram incluídos apenas os alunos autoclassificados como brancos, pretos ou pardos, uma vez que o número de alunos amarelos e indígenas é pequeno.

\section{MODELO E VARIÁVEIS}

A proficiência em Matemática, transformada em variável discreta de três níveis de desempenho, é a variável resposta neste trabalho.

Para criar os três níveis, utilizou-se como referência a interpretação pedagógica proposta por Soares (2009) aplicada à escala do Saeb, que é a mesma da Prova Brasil. O autor propõe a divisão da escala única de 500 pontos em quatro pontos de cortes e lhes atribui rótulos com claro significado normativo. São eles: nível insuficiente, básico, adequado e avançado. Esses níveis têm uma clara interpretação pedagógica, pois indicam necessidades específicas dos alunos em cada situação, em termos de reforço ou proposição de desafios.

\footnotetext{
O aluno classificado no nível Adequado demonstra dominar os conteúdos e habilidades esperados para o seu estágio escolar. Os do nível Avançado dominam a competência de forma especialmente completa, ultrapassando o esperado para o seu estágio escolar. O nível Básico congrega os alunos que demonstram domínio apenas parcial da competência. Finalmente, os alunos do nível Insuficiente mostram domínio rudimentar da competência medida (SOARES, 2009).
} 
Atualmente observa-se percentual muito baixo de alunos no nível avançado. Por isso, trabalhou-se com apenas três níveis: insuficiente, básico e adequado/avançado, este último constituído pela junção dos dois últimos níveis. Como consequência, assume-se que não há diferença conceitual entre desempenhos que estão nos níveis adequado e avançado, ainda que as diferenças possam impactar, por exemplo, em situações de seleção.

A análise consistiu na estimação, via HLM 7.01, de quatro modelos multinomiais hierárquicos (RAUDENBUSH et al., 2011). Nos modelos, o nível básico foi tomado como categoria de referência. Assim estimaram-se: as chances de um aluno estar no nível insuficiente, comparado às chances de estar no nível básico; e as chances de um aluno estar no nível adequado, comparado às chances de estar no nível básico.

Como o objetivo desse trabalho era estimar o efeito de cada escola de educação básica em cada uma das três edições da Prova Brasil (2007, 2009 e 2011), foi necessária a criação de um novo identificador de escola que a distinguisse nas três edições. Como resultados desse procedimento, foram obtidos 161.444 novos códigos de escolas (pseudoescolas), entre os quais foram distribuídos os 12.660 .302 alunos. Chama-se de pseudoescola a unidade que possui função no modelo estatístico utilizado.

O efeito de cada escola corresponde ao acréscimo na probabilidade de se observar determinado nível de proficiência em função de o aluno estar matriculado em uma dada escola. A média da distribuição dos efeitos é fixada em zero, uma restrição necessária para o seu cálculo (RAUDENBUSH; WILLMS, 1995). Os alunos de escolas com efeitos negativos têm maior probabilidade de estarem no nível insuficiente ou no básico, do que no nível adequado. Como os efeitos das escolas nas diferentes edições foram estimados conjuntamente, os valores obtidos são comparáveis ao longo das três edições da Prova Brasil.

O primeiro modelo não contém variáveis no nível 2 (equação do modelo 1, no Apêndice A). Assim, os efeitos estimados não consideram a composição social e econômica das escolas, sendo incluídas apenas as variáveis referentes às 
7 Para a discussão sobre a classificação de raça e cor no Brasil, ver Osório (2003) características dos alunos (nível 1): sexo, cor, atraso escolar e nível socioeconômico, variáveis fora do controle imediato da escola. Também incluiu-se uma variável indicadora do ano escolar, $5^{\circ}$ ou $9^{\circ}$, que o aluno frequentava quando da realização do teste. A rigor, os efeitos estimados não constituiriam o efeito-escola, pois não consideraram outras características do contexto que também estão fora do controle da escola. Contudo, a estimação dos efeitos pelo modelo 1 objetivou compreender como estes estão associados à composição socioeconômica, racial e por gênero dos estabelecimentos.

O nível socioeconômico (NSE) do aluno foi medido por meio da síntese, em uma única medida, de vários itens do questionário contextual, respondido pelos estudantes, sobre escolaridade e setor ocupacional de seus pais, posse de vários itens de conforto e contratação de empregados domésticos no domicílio. Os detalhes sobre o cálculo do NSE estão em Alves, Soares e Xavier (2014).

$\mathrm{O}$ atraso escolar mede a diferença entre a idade do aluno e a idade esperada para o ano escolar em que ele se encontrava, quando da realização do teste.

Foram considerados três tipos de composição escolar: composição por nível socioeconômico; composição racial; e composição por gênero.

A primeira é a média do nível socioeconômico dos alunos por escola, valor obtido no mesmo estudo de Alves, Soares e Xavier (2014). A composição racial é dada pela proporção alunos pretos ${ }^{7}$ por escola e a composição por gênero é obtida pela proporção de meninas por escola.

Além das variáveis de nível 1 descritas anteriormente, o modelo 2 inclui, como variável de controle de nível 2, o nível socioeconômico médio da escola (equação do modelo 2, no Apêndice A). Como resultado do ajuste desse modelo, foi possível observar a distribuição dos efeitos, pela composição racial e por gênero do alunado, independentemente do contexto socioeconômico dos estabelecimentos.

O terceiro modelo teve como objetivo analisar o eventual impacto da composição social e econômica das escolas (equação do modelo 3, no Apêndice A). Para isso, o modelo utilizado inclui, como variáveis de controle, as mesmas 
empregadas no segundo modelo e também a proporção de alunos pretos por escola, além da infraestrutura das escolas.

Também, a partir desse modelo, foram estimados os efeitos das escolas. Estes podem ser entendidos como valores que representam mais rigorosamente o efeito-escola pelo motivo de retratarem o que as escolas agregam aos seus alunos, considerando-se suas características individuais e contextuais.

A medida de infraestrutura foi desenvolvida a partir das informações do questionário contextual da Prova Brasil (itens observados pelo aplicador do teste) e das informações que constam do Censo Escolar de 2011 sobre instalações e recursos disponíveis. Foi calculado um indicador utilizando-se um modelo de Teoria da Resposta ao Item (TRI) para respostas graduadas. A escala original em desvios padrão foi transformada numa escala de 0 a 10 pontos.

O objetivo do quarto modelo foi estimar o impacto do nível socioeconômico das escolas sobre o efeito da raça e do sexo do aluno sobre o seu desempenho (equação do modelo 4, no Apêndice A). Ou seja, o interesse está na interação entre essas variáveis.

As variáveis incluídas nos diferentes modelos podem ser visualizadas nos quadros 1 e 2 a seguir.

QUADRO 1 - Variáveis incluídas no nível 1 - nível dos alunos

\begin{tabular}{|c|c|c|c|}
\hline VARIÁVEL* & TIPO & DESCRIÇÃO & CENTRALIZAÇÃO** \\
\hline Nível socioeconômico do aluno & Contínua & Escala de 0 a 10 & Na grande média*** \\
\hline Série & Binária & $1=9^{\circ}$ ano e $0=5^{\circ}$ ano & Não centralizada \\
\hline Sexo & Binária & $1=$ feminino e 0 = masculina & Não centralizada \\
\hline Missing para sexo & Binária & $1=$ missing e $0=$ outros valores & Não centralizada \\
\hline Brancos & Binária & $1=$ branco e $0=$ pardo & Não centralizada \\
\hline Pretos & Binária & $1=$ preto e 0 = pardo & Não centralizada \\
\hline Missing para raça & Binária & $1=$ missing e $0=$ outros valores & Não centralizada \\
\hline Atraso escolar & Binária & $\begin{array}{c}1=\text { um ano ou mais de atraso e } \\
0=\text { não possui atraso }\end{array}$ & Não centralizada \\
\hline Missing para atraso escolar & Binária & $1=$ missing e $0=$ outros valores & Não centralizada \\
\hline
\end{tabular}

* Nos quatro modelos são incluídas todas as variáveis descritas no quadro.

** Sobre centralização das variáveis nos dois níveis, ver Raudenbush e Bryk (2002).

*** Esta variável foi incluída no modelo centralizada na grande média, tendo em vista que é o nível do aluno numa hierarquia socioeconômica que interessa para explicar o seu desempenho e não sua posição relativa na escola em que estuda.

Fonte: Elaboração das autoras. 
QUADRO 2 - Variáveis incluídas no nível 2 - nível das escolas

\begin{tabular}{|l|c:c|c|c|}
\hline VARIÁVEL & TIPO & DESCRIÇÃO & CENTRALIZAÇÃO * \\
\hline $\begin{array}{l}\text { Nível socioeconômico } \\
\text { médio da escola** }\end{array}$ & Contínua & $\begin{array}{l}\text { Média do nível socioeconômico dos } \\
\text { alunos por escola - escala de O a 10 }\end{array}$ & Na grande média \\
\hline Infraestrutura*** & Contínua & Escala de O a 10 & Na grande média \\
\hline $\begin{array}{l}\text { Proporção de meninas na } \\
\text { escola }{ }^{* * *}\end{array}$ & Contínua & $\vdots$ & Escala de O a 1 & Na grande média \\
\hline $\begin{array}{l}\text { Proporção de alunos } \\
\text { pretos na escola }{ }^{* * *}\end{array}$ & Contínua & Escala de O a 1 & Na grande média \\
\hline
\end{tabular}

* Sobre centralização das variáveis nos dois níveis, ver Raudenbush e Bryk (2002).

** Variáveis não incluídas no modelo 1.

*** Variáveis não incluídas nos modelos 1 e 2.

Fonte: Elaboração das autoras.

\section{RESULTADOS E DISCUSSÃO}

Os efeitos das escolas são a expressão da parcela do desempenho acadêmico dos alunos que pode ser atribuída às práticas da escola, excluídas as características pessoais e familiares de seus alunos e também as do ambiente escolar que estão fora do controle da escola.

Como consequência do modelo de análise - um modelo hierárquico multinomial - dois efeitos para cada escola são produzidos. O efeito 1 traduz a capacidade de uma dada escola de levar o aluno do nível insuficiente para o básico. Pode-se dizer que esse efeito é a expressão da capacidade de uma escola de retirar seus alunos da exclusão.

O efeito 2 estima as chances de um indivíduo estar no nível adequado em relação a estar no nível básico. Esse efeito, por sua vez, deve ser interpretado como a capacidade da escola de garantir que seus alunos estejam preparados para dar continuidade aos estudos, ter uma trajetória escolar regular que lhes assegure uma vida adulta produtiva e o exercício da cidadania. Ou seja, esse efeito indica a capacidade de a escola garantir o direito à educação.

Ressalta-se que o nível básico, embora seja uma categoria entre o nível insuficiente e o adequado na escala ordinal adotada, não pode ser considerado um nível intermediário de aprendizagem. Um aluno nesse nível detém apenas domínio parcial da competência esperada para a série que está cursando. 
Valores negativos dos efeitos da primeira equação são desejáveis, uma vez que se espera que uma boa escola diminua as chances de seus alunos permanecerem no nível insuficiente. Já os efeitos negativos da segunda equação são indesejáveis, tendo em vista que não se espera que uma escola diminua as chances de seus alunos estarem no nível adequado. Para a simplicidade da apresentação, optou-se por colocar os efeitos 1 e 2 na mesma direção. Para tanto, os efeitos da primeira equação foram multiplicados por -1 .

Assim, no que se segue, as escolas com efeitos negativos ou iguais a zero indicam que as práticas internas, prevalentes no conjunto dessas escolas, não ajudam no aprendizado dos seus alunos, enquanto as que têm efeitos positivos são escolas que levam seus alunos além do que é esperado pelas suas características socioculturais.

\section{EFEITO DAS ESCOLAS AO LONGO DO TEMPO}

O Gráfico 1 apresenta os efeitos das escolas obtidos pelo modelo 1 . Observa-se que as médias dos efeitos 1 e 2 aumentam ao longo do tempo. Em 2011, as médias são positivas, indicando uma melhoria ao longo do tempo tanto na capacidade de as escolas retirarem seus alunos da exclusão (efeito 1), quanto na de promovê-los aos níveis adequados de aprendizagem (efeito 2). Entretanto, a melhoria desses efeitos pode refletir apenas diferenças entre o contexto socioeconômico das escolas, uma vez que o modelo 1 não inclui esse fator como controle. Para investigar essa relação pode-se verificar, na Tabela 1, a média dos efeitos estimados no modelo 1 pelos fatores contextuais das escolas. 
GRÁFICO 1 - Efeitos das escolas sem considerar a composição socioeconômica, racial e de gênero dos estabelecimentos, segundo a edição da Prova Brasil

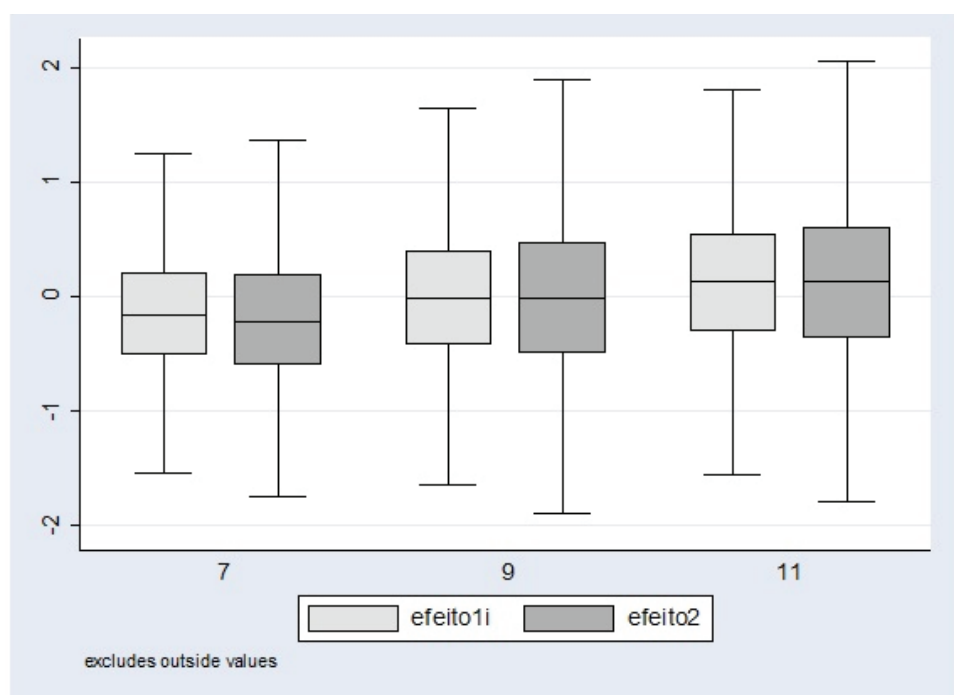

Fonte: Elaboração das autoras com base nos dados da Prova Brasil 2007, 2009 e 2011

Pela Tabela 1, nota-se que, quanto maior o nível socioeconômico das escolas, maiores são os efeitos produzidos por elas. Em outros termos, as escolas podem produzir bons resultados simplesmente porque trabalham em contexto mais favorável.

Em relação à proporção de alunos pretos na escola, a menor presença desse grupo é representada pelo quintil 1 e a maior corresponde ao quintil 5. Observa-se que, quanto maior a proporção de alunos pretos na escola, menores são os efeitos produzidos por ela (Tabela 1). Há duas hipóteses plausíveis para explicar tal resultado: i) a distribuição evidencia um componente de desvalorização e discriminação deste grupo no sistema escolar que aparece no nível do contexto da escola; e ii) não é possível falar de discriminação porque a distribuição observada para escolas com maior proporção de alunos pretos é resultante das desvantagens econômicas das escolas nas quais eles estudam.

Vale lembrar que o modelo 1 foi controlado pelas características dos alunos: seu nível socioeconômico, sua cor, seu sexo, sua trajetória escolar e a série que frequenta. Nesse modelo não havia controle de variáveis relacionadas ao contexto escolar. 
Em relação à proporção de meninas, quanto maior o número de meninas na escola, maiores são os efeitos produzidos, exceto no último quintil (Tabela 1). Uma suposição para esse resultado pode residir no fato de que, embora o maior número de meninas crie um ambiente disciplinar mais propício à aprendizagem, uma escola composta predominante por meninas, independentemente da composição socioeconômica da escola, pode não fazer diferença em termos de melhoria dos efeitos.

TABELA 1 - Média dos efeitos das escolas (Modelo 1) segundo os quintis do nível socioeconômico das escolas, da proporção de alunos pretos e de meninas por escola

\begin{tabular}{|c|c|c|c|c|c|c|}
\hline \multirow{2}{*}{$\begin{array}{l}\text { QUINTIS DA } \\
\text { DISTRIBUIÇÃO }\end{array}$} & \multicolumn{2}{|c|}{ NÍVEL SOCIOECONÔMICO } & \multicolumn{2}{|c|}{$\begin{array}{l}\text { PROPORÇÃO DE ALUNOS } \\
\text { PRETOS }\end{array}$} & \multicolumn{2}{|c|}{$\begin{array}{l}\text { PROPORÇÃO DE } \\
\text { MENINAS }\end{array}$} \\
\hline & EFEITO 1 & EFEITO 2 & EFEITO 1 & EFEITO 2 & EFEITO 1 & EFEITO 2 \\
\hline 1 & $-0,421$ & $-0,455$ & 0,152 & 0,173 & $-0,111$ & $-0,124$ \\
\hline 2 & $-0,188$ & $-0,225$ & 0,086 & 0,104 & $-0,001$ & 0,007 \\
\hline 3 & 0,050 & 0,035 & 0,005 & 0,012 & 0,050 & 0,062 \\
\hline 4 & 0,160 & 0,177 & $-0,083$ & $-0,090$ & 0,069 & 0,073 \\
\hline 5 & 0,399 & 0,467 & $-0,163$ & $-0,201$ & $-0,007$ & $-0,019$ \\
\hline
\end{tabular}

Fonte: Elaboração das autoras com base nos dados da Prova Brasil 2007, 2009 e 2011.

As hipóteses relacionadas à proporção de alunos pretos e de meninas podem ser verificadas quando se observa a distribuição dos efeitos calculados pelo modelo 2, o qual insere o nível socioeconômico das escolas como variável de controle (Tabela 2).

Verifica-se que o padrão de distribuição dos efeitos é muito semelhante àquele apresentado na Tabela 1. Entretanto, a magnitude dos efeitos na Tabela 2 é menor. É possível observar também que, ao controlar pela média do nível socioeconômico das escolas, os efeitos do último quintil da proporção de meninas por escola tornam-se positivos.

Assim, até agora, pode-se dizer que as desvantagens das escolas com maior número de alunos pretos não se devem ao nível socioeconômico desses grupos nem à média do nível socioeconômico das escolas. Esses fenômenos são independentes, 
como mostra a Tabela 2. Pode-se afirmar também que, independentemente do nível socioeconômico das escolas, o maior número de meninas tende a oferecer à escola maiores condições de produzir efeitos positivos tanto para a retirada de seus alunos da exclusão quanto para a promoção destes ao nível de aprendizagem adequado.

TABELA 2 - Média dos efeitos das escolas (Modelo 2) por quintis da proporção de alunos pretos e de meninas por escola

\begin{tabular}{c|c:c:c|c}
\hline \multirow{2}{*}{$\begin{array}{c}\text { QUINTIS DA } \\
\text { DISTRIBUIÇÃO }\end{array}$} & \multicolumn{2}{|c|}{$\begin{array}{c}\text { PROPORÇÃO DE } \\
\text { ALUNOS PRETOS }\end{array}$} & PROPORÇÃO DE MENINAS \\
\cline { 2 - 5 } & EFEITO 1 & EFEITO 2 & EFEITO 1 & EFEITO 2 \\
\hline 1 & 0,127 & 0,145 & $-0,052$ & $-0,057$ \\
\hline 2 & 0,057 & 0,071 & $-0,040$ & $-0,037$ \\
\hline 3 & $-0,010$ & $-0,004$ & $-0,007$ & $-0,001$ \\
\hline 4 & $-0,075$ & $-0,081$ & 0,034 & 0,034 \\
\hline 5 & $-0,101$ & $-0,132$ & 0,065 & 0,061 \\
\hline
\end{tabular}

Fonte: Elaboração das autoras com base nos dados da Prova Brasil 2007, 2009 e 2011.

Em síntese, a análise da distribuição dos efeitos das escolas indica que eles variam segundo a composição socioeconômica e social dos estabelecimentos. Portanto, para estimar o efeito-escola de modo mais rigoroso, é importante considerar o nível socioeconômico das escolas e a proporção de alunos pretos e de meninas por escola.

\section{FATORES ASSOCIADOS AO DESEMPENHO ESCOLAR}

O modelo 3 apresenta a medida do impacto de todos os fatores associados ao desempenho escolar usados como controles - sexo, cor, nível socioeconômico, atraso escolar do aluno e ano escolar que o aluno está cursando - e, também, a infraestrutura das escolas. Finalmente, o modelo inclui as variáveis explicativas - o nível socioeconômico das escolas e a proporção de alunos pretos e de meninas por escola - com o objetivo de obter uma medida de seu impacto. A Tabela 3 mostra a razão de chances por nível de desempenho. O nível básico é a categoria de referência.

Observa-se que, quanto maior o nível socioeconômico das escolas, menores são as chances de um aluno permanecer no 
nível insuficiente de aprendizagem e maiores as chances de um aluno estar no nível adequado. Ressalta-se que na população de escolas deste estudo não estão incluídas as escolas públicas federais nem as privadas.

Quanto maior a proporção de alunos pretos, independentemente de quaisquer outras características das escolas e de seus alunos controladas pelo modelo, maiores são as chances de um aluno permanecer no nível insuficiente e menores são as suas chances de passar para o nível adequado. Ou seja, qualquer que seja o aluno que estude numa escola com maior proporção de alunos pretos teria suas chances de estar no nível adequado diminuídas.

Já quanto maior a proporção de meninas, menores são as chances de um aluno permanecer no nível insuficiente e maiores são as suas chances de passar para o nível adequado. Isso quer dizer que, embora as meninas possuam desvantagens em relação aos meninos no desempenho de Matemática, o maior número delas na escola proporciona um ambiente acadêmico e disciplinar mais favorável à aprendizagem.

Por outro lado, quanto maior for a infraestrutura das escolas, menores serão as chances de um aluno permanecer no nível insuficiente e maiores serão suas chances de passar para o nível adequado.

Pelas variáveis do nível individual, alunos com nível socioeconômico mais alto, no $9^{\circ}$ ano, meninos, brancos e sem atraso escolar têm menor probabilidade de estarem no nível insuficiente. Alunos de nível socioeconômico mais alto e de cor branca têm maior chance de estarem no nível adequado de desempenho.

Observa-se, ainda, que a razão de chances de os alunos pretos permanecerem no nível insuficiente é maior que um, indicando que eles têm mais chance de estarem nesse nível quando comparados aos pardos. Adicionalmente, quando comparados aos pardos, eles têm menos chance de estarem no nível adequado. A desvantagem desse grupo social, independentemente de fatores socioeconômicos, também se manifesta em termos coletivos, quando se analisa a composição das escolas por cor. Alunos, sejam eles pretos ou não, quando estudam em escolas com uma concentração maior de alunos 
pretos, apresentam resultados piores do que se estudassem numa escola com maior concentração de brancos.

TABELA 3 - Razão de chances por nível de desempenho em Matemática - modelo de regressão do intercepto como resposta

\begin{tabular}{|c|c|c|}
\hline & \multicolumn{2}{|c|}{$\begin{array}{l}\text { RAZÃO DE CHANCES POR NÍVEL DE } \\
\text { DESEMPENHO EM MATEMÁTICA }\end{array}$} \\
\hline & INSUFICIENTE / BÁSICO & ADEQUADO / BÁSICO \\
\hline Intercepto, $\gamma_{00}$ & 0,503 & 0,717 \\
\hline Nível socioeconômico das escolas, $\gamma_{0 I}$ & 0,622 & 1,629 \\
\hline Proporção de pretos, $\gamma_{02}$ & 2,649 & 0,209 \\
\hline Proporção de meninas, $\gamma_{03}$ & 0,705 & 1,085 \\
\hline Infraestrutura, $\gamma_{04}$ & 0,933 & 1,119 \\
\hline Nível socioeconômico dos alunos, $\gamma_{10}$ & 0,938 & 1,143 \\
\hline Série, $\gamma_{20}$ & 0,898 & 0,271 \\
\hline Sexo, $\gamma_{30}$ & 1,168 & 0,764 \\
\hline Missing sexo, $\gamma_{40}$ & 1,908 & 0,510 \\
\hline Branco, $\gamma_{50}$ & 1,012 & 1,138 \\
\hline Preto, $\gamma_{60}$ & 1,251 & 0,721 \\
\hline Missing cor, $\gamma_{70}$ & 1,347 & 0,804 \\
\hline Atraso escolar, $\gamma_{80}$ & 1,778 & 0,516 \\
\hline Missing atraso escolar, $\gamma_{90}$ & 1,374 & 0,864 \\
\hline
\end{tabular}

Notas: 1) Categoria de referência: nível básico; 2) Todos os coeficientes são significativos ao nível de 0,01.

Fonte: Elaboração das autoras com base nos dados da Prova Brasil 2007, 2009 e 2011.

Os valores dos efeitos das escolas também foram estimados a partir do modelo 3, cuja evolução é apresentada no Gráfico 2. Observa-se que a tendência é um aumento da mediana dos efeitos de 2007 para 2011. Entretanto, esses valores possuem um intervalo menor do que aqueles verificados no Gráfico 1. Isso significa que há menor dispersão entre as escolas, ou seja, os mesmos tipos de efeitos (positivos ou negativos) têm magnitudes diferentes. 
GRÁFICO 2 - Efeitos das escolas considerando a composição socioeconômica, racial e de gênero dos estabelecimentos, segundo a edição da Prova Brasil

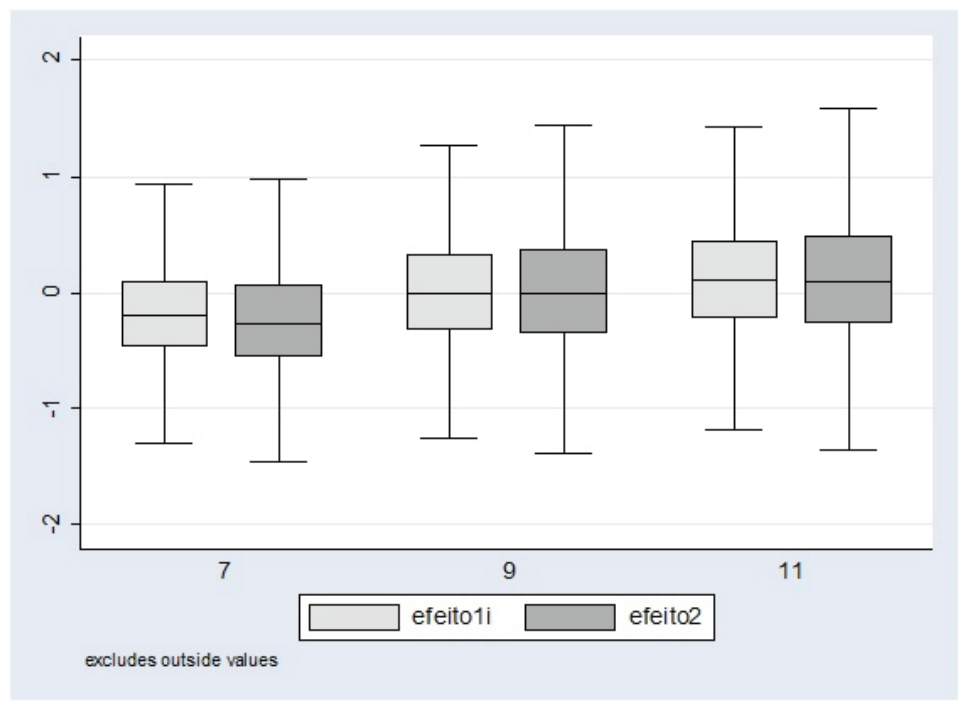

Fonte: Elaboração das autoras com base nos dados da Prova Brasil 2007, 2009 e 2011.

Para explorar um pouco mais as desvantagens de meninas e pretos de estarem em determinado nível de desempenho, o modelo 4 inclui a interação entre nível socioeconômico das escolas e sexo e cor. Este é um modelo cujos coeficientes de meninas, brancos e pretos se tornam a variável resposta no nível 2 do modelo hierárquico, sendo que, no nosso caso, o nível socioeconômico das escolas é a variável explicativa.

$\mathrm{O}$ aumento da média do nível socioeconômico da escola leva a três observações: redução das chances das meninas em permanecerem no nível insuficiente e aumento das chances delas em passarem para o nível adequado de Matemática; redução das chances dos alunos brancos em permanecerem no nível insuficiente e aumento das chances deles em passarem para o nível adequado de Matemática; e redução das chances dos alunos pretos em permanecerem no nível insuficiente, mas não aumentam as chances deles em passarem para o nível adequado de Matemática. 
TABELA 4 - Razão de chances por nível de desempenho em Matemática - modelo do intercepto e da inclinação como resposta

\begin{tabular}{|c|c|c|}
\hline & \multicolumn{2}{|c|}{$\begin{array}{l}\text { RAZÃO DE CHANCES POR NÍVEL DE DESEMPENHO EM } \\
\text { MATEMÁTICA }\end{array}$} \\
\hline & INSUFICIENTE / BÁSICO & ADEQUADO / BÁSICO \\
\hline Intercepto, $\gamma_{00}$ & 0,500 & 0,727 \\
\hline Nível socioeconômico das escolas, $\gamma_{0 l}$ & 0,698 & 1,576 \\
\hline Proporção de alunos pretos, $\gamma_{02}$ & 2,660 & 0,200 \\
\hline Proporção de meninas, $\gamma_{03}$ & 0,685 & 1,119 \\
\hline Infraestrutura, $\gamma_{04}$ & 0,932 & 1,120 \\
\hline Nivel socioeconômico dos alunos, $\gamma_{10}$ & 0,938 & 1,142 \\
\hline Série, $\gamma_{20}$ & 0,899 & 0,270 \\
\hline Sexo, $\gamma_{30}$ & 1,182 & 0,754 \\
\hline NSE das escolas * sexo, $\gamma_{3 I}$ & 0,856 & 1,014 \\
\hline Missing sexo, $\gamma_{40}$ & 1,915 & 0,510 \\
\hline Branco, $\gamma_{50}$ & 1,033 & 1,083 \\
\hline NSE das escolas ${ }^{*}$ branco, $\gamma_{51}$ & 0,868 & 1,115 \\
\hline Preto, $\gamma_{60}$ & 1,243 & 0,745 \\
\hline NSE das escolas ${ }^{*}$ preto, $\gamma_{61}$ & 1,067 & 0,910 \\
\hline Missing cor, $\gamma_{70}$ & 1,347 & 0,804 \\
\hline Atraso escolar, $\gamma_{80}$ & 1,779 & 0,516 \\
\hline Missing atraso escolar, $\gamma_{90}$ & 1,382 & 0,864 \\
\hline
\end{tabular}

Notas: 1) Categoria de referência: nível básico; 2) Todos os coeficientes são significativos ao nível de 0,01. Fonte: Elaboração das autoras com base nos dados da Prova Brasil 2007, 2009 e 2011.

\section{CONSIDERAÇÕES FINAIS}

O processo de escolarização é uma etapa do ciclo de vida dos indivíduos que não se resume apenas ao acesso e à progressão destes no sistema escolar, mas também envolve o aprendizado. A escolarização básica adequada influencia as possibilidades futuras de realização educacional superior, inserção ocupacional e rendimentos mais altos. O desempenho escolar adequado é um aspecto importante para a análise das desigualdades de resultados em geral.

Os resultados deste trabalho indicam como as escolas podem reproduzir as desigualdades sociais encontradas na sociedade em geral, que não se reduzem às diferenças socioeconômicas, refletindo, também, clivagens de raça e 
gênero. Esta pesquisa adotou uma abordagem alternativa para a análise do desempenho escolar que visa a explorar a dimensão das desigualdades, estimando os efeitos das escolas e os impactos da composição escolar por níveis de desempenho

Um primeiro conjunto de achados referentes à estimação do efeito das escolas indica que, mesmo considerando-se o nível socioeconômico das escolas, quanto maior a proporção de meninas numa escola, mais fácil será para esta produzir efeito para a retirada dos seus alunos da exclusão ou para promovê-los ao nível adequado.

Uma explicação possível para esse resultado é o clima acadêmico possibilitado pela maior presença de meninas, pois estas, supostamente, estariam mais propensas a um comportamento esperado de bom aluno. Esse fato merece investigações futuras.

Há poucos estudos que exploram a composição racial e por gênero das escolas e os seus efeitos sobre o desempenho acadêmico. Carvalho (2003) argumenta que as professoras avaliam como bons alunos crianças participativas e críticas, independentemente do gênero. Entretanto, a pesquisa da autora não se concentra na investigação do clima acadêmico da escola, mas sim nos resultados individuais.

No Brasil, as alunas entram na escola mais cedo, têm trajetória escolar mais regular e permanecem mais tempo na escola em comparação aos meninos. A maior "feminização" da escola parece ter um efeito positivo para o coletivo, aumentando o efeito da escola. Entretanto, as vantagens das meninas na educação não se refletem futuramente no mercado de trabalho.

Apesar de a participação da mulher no mercado de trabalho ser crescente, as ocupações tipicamente femininas têm menor prestígio e baixos retornos salariais. Não há dados nacionais disponíveis que possam ajudar na investigação entre o desempenho escolar e as escolhas das mulheres por carreiras mais competitivas. Nesta pesquisa também encontramos que as meninas possuem menos chances de alcançarem o aprendizado adequado em Matemática, fato que pode ajudar a entender a baixa escolha por carreiras mais competitivas, tais como Engenharia. 
Em relação à proporção de alunos pretos, a escola enfrenta mais dificuldades em produzir efeitos tanto para a retirada dos seus alunos da exclusão, quanto para a promoção destes ao nível de adequação.

Uma explicação plausível que justifique a presença do fator raça como inibidor de efeitos desejáveis pode residir nas expectativas quanto ao sucesso de alunos pretos, provocando um nivelamento por baixo. Carvalho (2004) argumenta que as concepções socialmente construídas de masculinidade e feminilidade, assim como de negritude e "branquitude”, estão também presentes na escola. Há uma tendência de professores associarem fracasso escolar, raça negra e masculinidade, ao mesmo tempo que as meninas são menos associadas a problemas escolares e tendem a ser "branqueadas", reforçando uma identidade dos meninos negros carregada de significados negativos: postura antiescolar; valorização da força física e da agressividade; e conquistas heterossexuais.

Um segundo conjunto de achados refere-se à estimação das chances de um aluno sair de uma situação de exclusão ou de permanecer no nível de adequação, considerando suas características individuais e escolares.

Observou-se que o aluno com maior nível socioeconômico e as meninas possuem probabilidade mais baixa de estarem no nível insuficiente de aprendizagem. Os alunos pretos têm mais chances de permanecerem no nível insuficiente.

Já os alunos com maior nível socioeconômico, os meninos e brancos são os que possuem mais chances de estarem no nível de aprendizagem adequado.

Quanto maiores os coeficientes relativos ao nível socioeconômico das escolas e à proporção de meninas, menores são as chances de os alunos permanecerem no nível insuficiente e maiores são as suas chances de estarem no nível adequado. E quanto maior a proporção de alunos pretos por escola, maiores são as chances de os alunos permanecerem no nível insuficiente e menores são as suas chances de estarem no nível adequado ou avançado.

Por fim, o aumento do nível socioeconômico das escolas diminui as chances de as meninas permanecerem no nível insuficiente e aumenta as suas chances de passarem para 
o nível adequado. O aumento do nível socioeconômico das escolas também diminui as chances de exclusão dos alunos pretos, mas não aumenta suas chances de passarem para o nível de adequação.

Observou-se, também, que a melhor infraestrutura da escola aumenta as chances de retirar o aluno do nível insuficiente e de estar no nível adequado. Entretanto, é preciso investigar mais os fatores escolares que contribuem para reduzir o efeito das características sociais e do contexto escolar nas chances de exclusão, sobretudo os relacionados à organização do trabalho pedagógico e aos processos de ensino e aprendizagem. Isso deverá ser buscado na continuidade deste programa de pesquisas sobre exclusão educacional.

\section{REFERÊNCIAS}

ALBERNAZ, Ângela; FERREIRA, Francisco H. G.; FRANCO, Creso. Qualidade e equidade no ensino fundamental brasileiro. Pesquisa e Planejamento Econômico, Rio de Janeiro, v. 32, n. 3, p. 453-476, 2005.

ALVES, Maria Teresa Gonzaga; FRANCO, Creso. A Pesquisa em eficácia escolar no Brasil. In: BROOKE, Nigel; SOARES, José Francisco (Org.). Pesquisa em eficácia escolar: origem e trajetórias. Belo Horizonte: Editora UFMG, 2008. p. 482-500.

ALVES, Maria Teresa Gonzaga; SOARES, José Francisco; XAVIER, Flavia Pereira. Índice socioeconômico das escolas de educação básica brasileiras. Ensaio: Avaliação e Políticas Públicas em Educação, Rio de Janeiro, v. 22, n. 84, p. 671-703, 2014.

ANDRADE, Josemberg M. de; LAROS, Jacob A. Fatores associados ao desempenho escolar: estudo multinível com dados do Saeb/2001. Psicologia: Teoria e Pesquisa, Brasília, v. 23, n. 1, p. 33-42, 2007.

ANDRADE, Renato Júdice de; SOARES, José Francisco. O efeito da escola básica brasileira. Estudos em Avaliação Educacional, São Paulo, v. 19, n. 41, p. 379-406, 2008.

BARBOSA, Maria Eugênia Ferrão; FERNANDES, Cristiano. A escola brasileira faz diferença? Uma investigação dos efeitos da escola na proficiência em Matemática dos alunos da $4^{\mathrm{a}}$ série. In: FRANCO, Creso (Ed.). Promoção, ciclos e avaliação educacional. Porto Alegre: ArtMed, 2001. p. 155-172.

BOURDIEU, Pierre. Classificação, desclassificação, reclassificação. In: NOGUEIRA, Maria Alice; CATANI, Afrânio (Ed.). Escritos de educação. Petrópolis: Vozes, 1998. p. 145-183.

BOURDIEU, Pierre; PASSERON, Jean-Claude. A reprodução: elementos para uma teoria do sistema de ensino. Rio de Janeiro: Francisco Alves, 1975. 
BROOKE, Nigel; CUNHA, Maria Amália de A. A avaliação externa como instrumento da gestão educacional nos estados. Estudos \& Pesquisas Educacionais, São Paulo, n. 2, p. 17-79, 2011.

BROOKE, Nigel; SOARES, José Francisco (Org.). Pesquisa em eficácia escolar: origem e trajetórias. Belo Horizonte: Editora UFMG, 2008.

CARVALHO, Maria Pinto de. Sucesso e fracasso escolar: uma questão de gênero. Educação e Pesquisa, São Paulo, v. 29, n. 1, p.185-193, jun. 2003.

O fracasso escolar de meninos e meninas: articulações entre gênero e cor/raça. Cadernos Pagu, Campinas, n. 22, p. 247-290, jun. 2004.

COLEMAN, James S. et al. Equality of educational opportunity. Washington, D.C.: US Government Printing Office, 1966.

DAVIS, Kingsley; MOORE, Wilbert E. Alguns princípios da estratificação social. In: VELHO, Otávio et al. (Org.). Estrutura de classes e estratificação social. Rio de Janeiro: Zahar, 1981. p. 114-132.

FLETCHER, Philip. À procura do ensino eficaz: relatório de pesquisa. Rio de Janeiro, PNUD/MEC/Saeb, 1997.

HALLER, Archibald O.; SARAIVA, Helcio U. Ascription and status transmission in Brazil. In: SCOVILLE, James G. Status influences in the Third World labor market: caste, gender and custom. New York: Walter de Gruyter, 1991. p. 63-93.

LEE, Valerie E. Using multilevel methods to investigate research questions that involve nested data. Estudos em Avaliação Educacional, São Paulo, n. 24, p. 46-68, 2001.

MORTIMORE, Peter; SAMMONS, Pamela; STOLL, Louise; LEWIS, David; ECOB, Russel. A importância da escola. In: BROOKE, Nigel; SOARES, José Francisco (Org.). Pesquisa em eficácia escolar: origem e trajetórias. Belo Horizonte: Editora UFMG, 2008.

OSÓRIO, Rafael Guerreiro. 0 sistema classificatório de "cor ou raça" do IBGE. Rio de Janeiro: Ipea, 2003 (Texto para discussão, n. 996).

PARSONS, Talcott. Equality and inequality in modern society, or social stratification revisited. Sociology Inquiry, v. 40, n. 2, p. 13-72, Apr. 1970. . 0 sistema das sociedades modernas. São Paulo: Pioneira, 1974.

RAUDENBUSH, Stephen W.; BRYK, Anthony S. Hierarchical linear models: applications and data analysis methods. 2. ed. Newbury Park, California: Sage, 2002.

RAUDENBUSH, Stephen. W.; WILLMS, Jon Douglas. The estimation of school effects. Journal of Educational and Behavioral Statistics, v. 20, n. 4, p. 307-335, 1995.

RAUDENBUSH, Stephen W. et al. HLM 7: Hierarchical linear and nonlinear modeling. Lincolnwood, IL: Scientific Software International, 2011.

RUMBERGER, Russell W.; PALARDY, Gregory J. Does segregation still matter? The impact of student composition on academic achievement in high school. Teachers College Record, v. 107, n. 9, p. 1999-2045, Sep. 2005. 
RUTTER, Michael et al. Fifteen thousand hours: secondary schools and their effects on children. Somerst: Open Books, 1979.

SOARES, José Francisco. Índice de Desenvolvimento da Educação de São Paulo - IDESP: bases metodológicas. São Paulo Perspectivas, São Paulo, v. 23, n. 1, p. 29-41, 2009.

SOARES, José Francisco; ALVES, Maria Teresa Gonzaga. Desigualdades raciais no sistema brasileiro de educação básica. Educação e Pesquisa, São Paulo, v. 29, n. 1, p. 147-165, 2003.

SOARES, José Francisco; CÉSAR, Cibele Comini; MAMBRINI, Juliana. Determinantes de desempenho dos alunos do ensino básico brasileiro: evidências do SAEB de 1997. In: FRANCO, Creso (Org.). Promoção, ciclos e avaliação educacional. Porto Alegre: ArtMed, 2001. p. 121-153.

SOARES, José Francisco; MAROTTA, Luana Desigualdades no sistema de ensino fundamental brasileiro. In: VELOSO, Fernando et al. (Org.). Educação básica no Brasil: construindo o país do futuro. Rio de Janeiro: Campus-Elsevier, 2009. p. 73-93.

SOARES, José Francisco et al. Exclusão intraescolar nas escolas públicas brasileiras: um estudo com dados da Prova Brasil 2005, 2007 e 2009. Brasília: Unesco, 2012. (Série Debates ED).

WILLMS, J. Douglas. Monitoring school performance: a guide for educators. Washington, D.C.: Falmer, 1992.

FLAVIA PEREIRA XAVIER

Professora Adjunta do Departamento de Ciências Aplicadas à Educação da Faculdade de Educação da Universidade Federal de Minas Gerais (DECAE/FaE/UFMG), Belo Horizonte, Minas Gerais, Brasil

flaviapx@ufmg.br

MARIA TERESA GONZAGA ALVES

Professora Adjunta do Departamento de Ciências Aplicadas à Educação da Faculdade de Educação da Universidade Federal de Minas Gerais (DECAE/FaE/UFMG), Belo Horizonte, Minas Gerais, Brasil

mtga@ufmg.br 


\section{APÊNDICE A: EQUAÇÕES DOS MODELOS HIERÁRQUICOS MULTINOMIAIS}

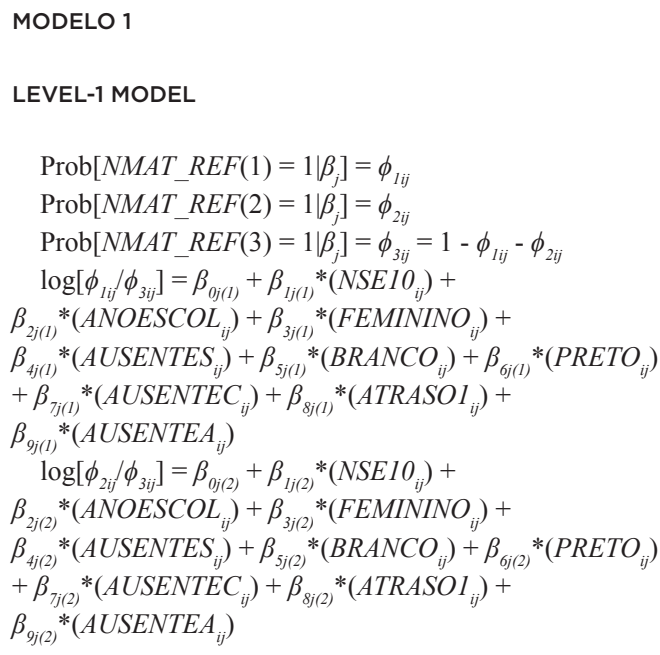

LEVEL-2 MODEL

$$
\begin{aligned}
& \beta_{0(I)}=\gamma_{00(I)}+u_{0 j(I)} \\
& \beta_{1(I)}=\gamma_{10(I)} ; \\
& \beta_{2(I)}=\gamma_{20(I)} \\
& \beta_{3(I)}=\gamma_{30(I)} \\
& \beta_{4(I)}=\gamma_{40(I)} \\
& \beta_{5(l)}=\gamma_{50(I)} \\
& \beta_{6(l)}=\gamma_{60(I)} \\
& \beta_{7(l)}=\gamma_{70(I)} \\
& \beta_{8(I)}=\gamma_{80(I)} \\
& \beta_{9(I)}=\gamma_{90(I)} \\
& \beta_{0(2)}=\gamma_{00(2)}+u_{0 j(2)} \\
& \beta_{1(2)}=\gamma_{10(2)} \\
& \beta_{2(2)}=\gamma_{20(2)} \\
& \beta_{3(2)}=\gamma_{30(2)} \\
& \beta_{4(2)}=\gamma_{40(2)} \\
& \beta_{5(2)}=\gamma_{50(2)} \\
& \beta_{6(2)}=\gamma_{60(2)} \\
& \beta_{7(2)}=\gamma_{70(2)} \\
& \beta_{8(2)}=\gamma_{80(2)} \\
& \beta_{9(2)}=\gamma_{90(2)}
\end{aligned}
$$

MODELO 2

LEVEL-1 MODEL

$$
\operatorname{Prob}\left[N M A T_{-} R E F(1)=1 \mid \beta_{j}\right]=\phi_{l i j}
$$$$
\operatorname{Prob}\left[N M A T_{-} R E F(2)=1 \mid \beta_{j}\right]=\phi_{2 i j}
$$$$
\operatorname{Prob}\left[N M A T \_R E F(3)=1 \mid \beta_{j}\right]=\phi_{3 i j}=1-\phi_{1 i j}-\phi_{2 i j}
$$$$
\log \left[\phi_{I i j} / \phi_{3 i j}\right]=\beta_{0 j(I)}+\beta_{I j(I)} *\left(N S E 10_{i j}\right)+
$$$$
\beta_{2 j(I)} *(A N O E S C O L)+\beta_{3 j(l)} *\left(\text { FEMININO }_{i j}\right)+
$$

$\beta_{4 j(1)} *\left(\right.$ AUSENTES $\left._{i j}\right)+\beta_{5 j(I)} *\left(\right.$ BRANCO $\left._{i j}\right)+\beta_{6 j(I)} *\left(\right.$ PRETO $\left._{i j}\right)$

$+\beta_{7 j(1)} *\left(\right.$ AUSENTEC $\left._{i j}\right)+\beta_{8 j(I)} *\left(\right.$ ATRASO1 $\left._{i j}\right)+$

$\beta_{9 j(I)} *\left(\right.$ AUSENTEA $\left.A_{i j}\right)$

$\log \left[\phi_{2 i j} / \phi_{3 i j}\right]=\beta_{0 j(2)}+\beta_{l j(2)} *\left(N S E 10_{i j}\right)+$

$\beta_{2 j(2)} *(A N O E S C O L)+\beta_{3 j(2)} *\left(\right.$ FEMININO $\left._{i j}\right)+$

$\beta_{4 j(2)} *\left(\right.$ AUSENTES $\left._{i j}\right)+\beta_{5 j(2)} *\left(\right.$ BRANCO $\left._{i j}\right)+\beta_{6 j(2)} *\left(\right.$ PRETO $\left._{i j}\right)$

$+\beta_{7 j(2)} *\left(\right.$ AUSENTEC $\left.C_{i j}\right)+\beta_{8 j(2)} *\left(\right.$ ATRASO1 $\left._{i j}\right)+$

$\beta_{9 j(2)} *\left(\right.$ AUSENTEA $\left.A_{i j}\right)$

LEVEL-2 MODEL

$$
\begin{aligned}
& \beta_{0(1)}=\gamma_{00(1)}+\gamma_{01(1)} *\left(M N S E_{-} F I R_{j}\right)+u_{0 j(1)} \\
& \beta_{1(1)}=\gamma_{10(1)} \\
& \beta_{2(l)}=\gamma_{20(1)} \\
& \beta_{3(1)}=\gamma_{30(1)} \\
& \beta_{4(1)}=\gamma_{40(1)} \\
& \beta_{5(1)}=\gamma_{50(1)} \\
& \beta_{6(1)}=\gamma_{60(1)} \\
& \beta_{7(1)}=\gamma_{70(1)} \\
& \beta_{8(1)}=\gamma_{80(1)} \\
& \beta_{9(1)}=\gamma_{90(1)} \\
& \beta_{0(2)}=\gamma_{00(2)}+\gamma_{01(2)} *\left(M N S E_{-} F I R_{j}\right)+u_{0 j(2)} \\
& \beta_{1(2)}=\gamma_{10(2)} \\
& \beta_{2(2)}=\gamma_{20(2)} \\
& \beta_{3(2)}=\gamma_{30(2)} \\
& \beta_{4(2)}=\gamma_{40(2)} \\
& \beta_{5(2)}=\gamma_{50(2)} \\
& \beta_{6(2)}=\gamma_{60(2)} \\
& \beta_{7(2)}=\gamma_{70(2)} \\
& \beta_{8(2)}=\gamma_{80(2)} \\
& \beta_{9(2)}=\gamma_{90(2)}
\end{aligned}
$$


(continuação)

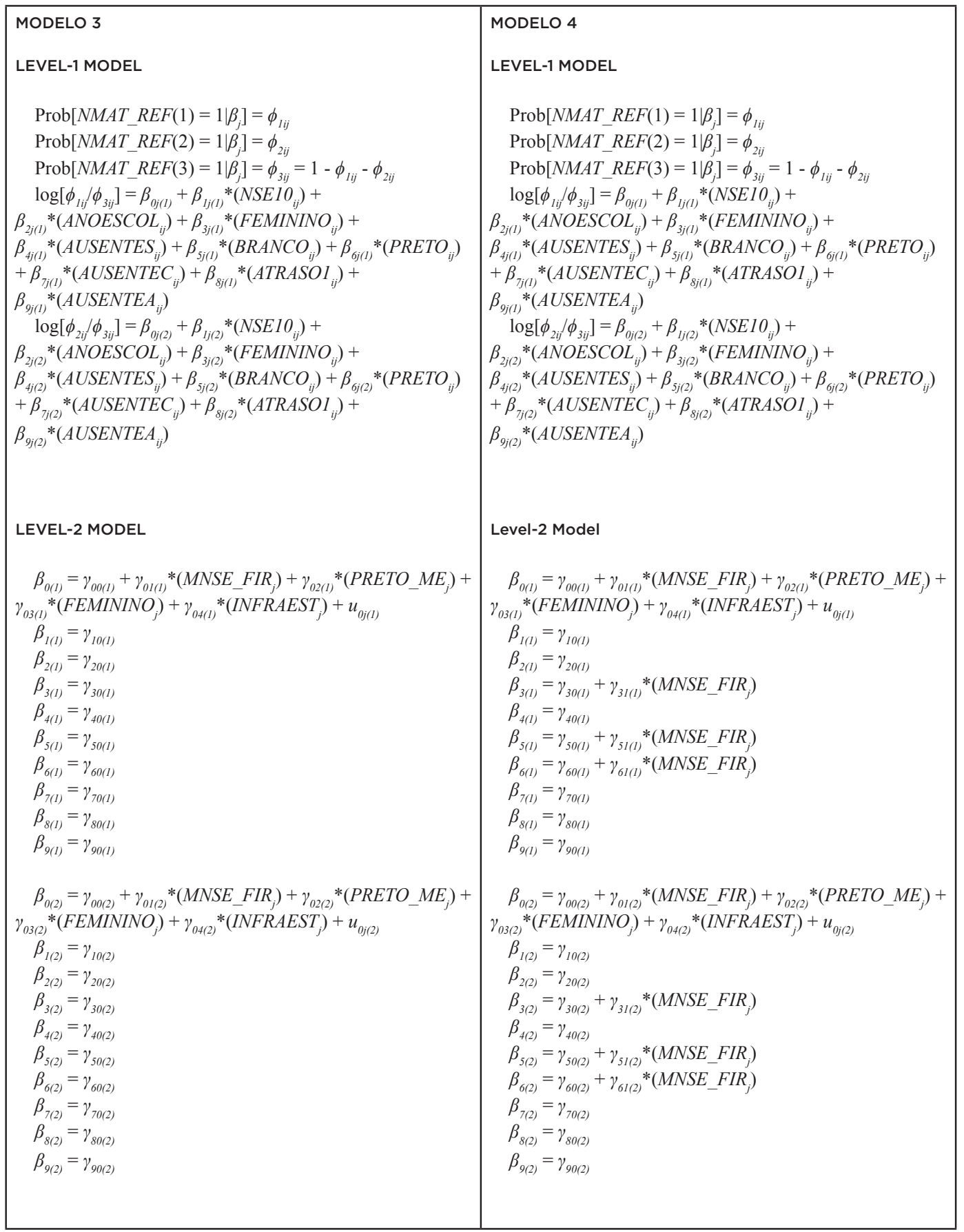

Recebido em: SETEMBRO 2014

Aprovado para publicação em: FEVEREIRO 2015 